УДК 517.98

F. JAWAD

\title{
NOTE ON SEPARATELY SYMMETRIC POLYNOMIALS ON THE CARTESIAN PRODUCT OF $\ell_{1}$
}

\begin{abstract}
F. Jawad. Note on separately symmetric polynomials on the Cartesian product of $\ell_{1}$, Mat. Stud. 50 (2018), 204-210.

In the paper, we describe algebraic bases in algebras of separately symmetric polynomials which are defined on Cartesian products of $n$ copies of $\ell_{1}$. Also, we describe spectra of algebras of entire functions, generated by these polynomials as Cartesian products of spectra of algebras of symmetric analytic functions of bounded type on $\ell_{1}$. Finally, we consider algebras of separately symmetric analytic functions of bounded type on infinite direct sums of copies of $\ell_{1}$. In particular, we show that there is a homomorphism from such algebra onto the algebra of all analytic functions of bounded type on a Banach space $X$ with an unconditional basis.
\end{abstract}

1. Introduction and preliminaries. Let $X$ be a complex Banach space. A function $f: X \rightarrow \mathbb{C}$ is called $G$-analytic if its restriction to any finite dimensional subspace of $X$ is analytic. A continuous $G$-analytic function is called analytic on $X$ or entire. If an entire function is bounded on all bounded subsets, then it is called a function of bounded type. An analytic function $P_{n}$ is $n$-homogeneous polynomial if $P_{n}(\lambda x)=\lambda^{n} P_{n}(x), x \in X, \lambda \in \mathbb{C}$. 0 -homogeneous polynomial is just a constant. A finite sum of homogeneous polynomials is a polynomial. It is well known that any entire function $f$ of bounded type can be represented by the series

$$
f(x)=\sum_{n=0}^{\infty} P_{n}(x),
$$

where $P_{n}$ are $n$-homogeneous polynomials and the series converges uniformly on all bounded subsets. On the other hand, every series (1) defines an entire function of bounded type if and only if the radius $\varrho_{0}(f)$ of convergence at zero is equal to infinity, where

$$
\varrho_{0}(f)=\left(\limsup _{n \rightarrow \infty}\left\|P_{n}\right\|^{1 / n}\right)^{-1} \text {. }
$$

For the general theory of analytic functions on Banach spaces we refer the reader to [9], [17].

Symmetric (invariant) polynomials and analytic functions with respect to actions of a group of operators on an infinite dimensional Banach space $X$ were investigated in many papers (see e.g. [3], [4], [8]). Uniform algebras of symmetric analytic functions on $\ell_{p}$ and their

2010 Mathematics Subject Classification: 46J15, 46J20.

Keywords: polynomials and analytic functions on Banach spaces; symmetric analytic functions; blocksymmetric polynomials; separately symmetric polynomials.

doi: $10.15330 / \mathrm{ms} .50 .2 .204-210$

(C) F. Jawad, 2018 
spectra were investigated in [1]. Topological and algebraic structures on spectra of algebras of symmetric entire functions of bounded type on $\ell_{p}$ were studied in [5]-[7] and on $L_{\infty}$ in [10], [11], [18]. Note that for different representations of a given group we can have different algebras of all symmetric polynomials.

Let $S$ be the group of all bijections of natural numbers $\mathbb{N}$. For every $\sigma \in S$ we define an action of $\sigma$ on $\ell_{1}$ by

$$
\sigma(x)=\left(x_{\sigma(1)}, x_{\sigma(2)}, \ldots\right),
$$

where $x=\left(x_{1}, x_{2}, \ldots\right) \in \ell_{1}$. A polynomial $P: \ell_{1} \rightarrow \mathbb{C}$ is said to be symmetric if $P(\sigma(x))=$ $P(x)$ for every $X \in \ell_{1}$ and permutation $\sigma \in S$. It is known [8] that polynomials

$$
F_{k}(X)=\sum_{n=1}^{\infty} x_{n}^{k}, \quad k=1,2, \ldots
$$

form an algebraic basis in the algebra of all symmetric polynomials $\mathcal{P}_{s}\left(\ell_{1}\right)$.

In the paper, we consider algebras of separately symmetric polynomials on the Cartesian product of a finite number of copies of $\ell_{1}, \underbrace{\ell_{1} \times \cdots \times \ell_{1}}_{n}$ and describe algebraic bases of these algebras. Also, we consider algebras of separately symmetric analytic functions of bounded type on infinite direct sums of copies of $\ell_{1}$.

Let us recall that a sequence of polynomials $\left\{P_{1}, P_{2}, \ldots, P_{n}, \ldots\right\}$ on a complex linear space $X$ is algebraically independent if for every $m \in \mathbb{N}$, for every polynomial $q: \mathbb{C}^{m} \rightarrow \mathbb{C}$ and every positive integer $i_{1}<\ldots<i_{m}$ the identity

$$
q\left(P_{i_{1}}(x), \ldots, P_{i_{m}}(x)\right)=0 \quad \forall x \in X
$$

implies that $q(z)=0 \forall z \in \mathbb{C}^{n}$. A sequence of polynomials on $X$ forms an algebraic basis in an algebra $\mathcal{P}$ of polynomials on $X$ if it is algebraically independent and generates this algebra.

2. Main results. Let us denote by

$$
\ell_{1}^{(n)}=\underbrace{\ell_{1} \times \cdots \times \ell_{1}}_{n}
$$

the Cartesian product of $n$ copies of $\ell_{1}$ with

$$
\|x\|=\left\|x^{(1)}\right\|+\cdots+\left\|x^{(n)}\right\|, \quad \text { where } \quad x=\left(x^{(1)}, \ldots, x^{(n)}\right) \in \ell_{1}^{(n)} .
$$

Definition 1. A polynomial $P: \ell_{1}^{(n)} \rightarrow \mathbb{C}$ is separately symmetric if for all permutations $\sigma_{1}, \ldots, \sigma_{n}$ on $\mathbb{N}$

$$
P\left(\sigma_{1}\left(x^{(1)}\right), \ldots, \sigma_{n}\left(x^{(n)}\right)\right)=P\left(x^{(1)}, \ldots, x^{(n)}\right) .
$$

Definition 2. A polynomial $P: \ell_{1}^{(n)} \rightarrow \mathbb{C}$ is a block-symmetric or MacMahon symmetric polynomial if for every permutation $\sigma$ on $\mathbb{N}$

$$
P\left(\sigma\left(x^{(1)}\right), \ldots, \sigma\left(x^{(n)}\right)\right)=P\left(x^{(1)}, \ldots, x^{(n)}\right) .
$$


We denote by $\mathcal{P}_{s s}\left(\ell_{1}^{(n)}\right)$ the algebra of all separately symmetrical polynomials on $\ell_{1}^{(n)}$. Note that $\ell_{1}^{(n)}$ is isometrically isomorphic to $\ell_{1}$. Indeed, the following map defined by

$$
I^{(n)}\left(x^{(1)}, \ldots, x^{(n)}\right)=\left(x_{1}^{(1)}, \ldots, x_{1}^{(n)}, x_{2}^{(1)}, \ldots, x_{2}^{(n)}, \ldots, x_{k}^{(1)}, \ldots, x_{k}^{(n)}, \ldots\right)
$$

is an isomorphism from $\ell_{1}^{(n)}$ onto $\ell_{1}$. If $P$ is a symmetric polynomial on $\ell_{1}$, then $P \circ I^{(n)}$ is separately symmetrical on $\ell_{1}^{(n)}$. So the map $P \mapsto P \circ I^{(n)}$ is a homomorphism of the algebra of all symmetric polynomials on $\ell_{1}, \mathcal{P}_{s}\left(\ell_{1}\right)$ to $\mathcal{P}_{s s}\left(\ell_{1}^{(n)}\right)$. Note that it is not onto. For example, $Q(x, y)=\sum_{i<j} x_{i} x_{j}+\sum_{i<j} y_{i} y_{j}$ is separately symmetric on $\ell_{1}^{(2)}$ but polynomial $P$ on $\ell_{1}$ defined by $P\left(x_{1}, y_{1}, \ldots, x_{n}, y_{n}, \ldots\right)=Q(x, y)$ is not symmetric.

Clearly, every separately symmetric polynomial is a block-symmetric polynomials. General algebraic properties of block-symmetric polynomials of finite number of variables can be found in [12]. Algebras generated by block-symmetric polynomials on $\ell_{1}$ and their spectra were investigated in [13], [14], [15].

We will say that $x \sim y$ for some $x, y \in \ell_{1}^{(n)}$ if $P(x)=P(y)$ for every $P \in \mathcal{P}_{s s}\left(\ell_{1}^{(n)}\right)$. For a given $x \in \ell_{1}^{(n)}$ and $1 \leq j \leq n$ we denote the support of $x^{(j)}$ by

$$
\operatorname{supp}_{j}(x)=\operatorname{supp}\left(x^{(j)}\right)=\left\{m \in \mathbb{N}: x_{m}^{(j)} \neq 0\right\} .
$$

Lemma 1. For every $x \in \ell_{1}^{(n)}$ there is $y \sim x \operatorname{such}$ that $\operatorname{supp}_{i}(y) \cap \operatorname{supp}_{j}(y)=\varnothing$ for all $1 \leq i<j \leq n$.

Proof. We observe, first that if $z \in \ell_{1}^{(n)}$ such that for some $1 \leq l \leq n$,

$$
z^{(l)}=\left(0, x_{1}^{(l)}, x_{2}^{(l)}, \ldots, x_{k}^{(l)}, \ldots\right)
$$

and $z^{(j)}=x^{(j)}$ for all $j \neq l$, then $z \sim x$. Indeed, let $P \in \mathcal{P}_{s s}\left(\ell_{1}^{(n)}\right)$. Let us denote by $P_{l}\left(z^{(l)}\right)$ the polynomial $P(z)$ for fixed $z^{(j)}, j \neq l$. Then $P_{l}$ is a symmetric polynomial on $\ell_{1}$ and as it is well known for symmetric polynomials,

$$
P_{l}\left(z^{(l)}\right)=P_{l}\left(0, x_{1}^{(l)}, x_{2}^{(l)}, \ldots, x_{k}^{(l)}, \ldots\right)=P_{l}\left(x_{1}^{(l)}, x_{2}^{(l)}, \ldots, x_{k}^{(l)}, \ldots\right)=P_{l}\left(x^{(l)}\right) .
$$

So $P(z)=P(x)$.

Now, it is enough to set

$$
y^{(j)}=(\underbrace{0, \ldots, 0}_{j-1}, x_{1}^{(j)}, \underbrace{0, \ldots, 0}_{n-1}, x_{2}^{(j)}, \ldots, \underbrace{0, \ldots, 0}_{n-1}, x_{k}^{(j)}, \ldots), \quad j=1,2, \ldots, n .
$$

Theorem 1. Polynomials

$$
F_{k}^{(j)}(x)=F_{k}\left(x^{(j)}\right)=\sum_{m=1}^{\infty}\left(x_{m}^{(j)}\right)^{k}, \quad j=1, \ldots, n, \quad k=1,2, \ldots
$$

form an algebraic basis in $\mathcal{P}_{s s}\left(\ell_{1}^{(n)}\right)$. 
Proof. As we observed, any separately symmetric polynomial $P$ is a block-symmetric polynomial and so it can be represented by the following algebraic basis in the algebra of blocksymmetric polynomials on $\ell_{1}$ (see $[15]$ ):

$$
F_{k_{1} \ldots k_{n}}(x)=\sum_{m=1}^{\infty}\left(x_{m}^{(1)}\right)^{k_{1}} \cdots\left(x_{m}^{(n)}\right)^{k_{n}}, \quad k_{1}, \ldots k_{n} \in \mathbb{Z}_{+} .
$$

Note that polynomials in (4) are separately symmetric only if just one nonnegative integer $k_{1}, \ldots, k_{n}$ is greater than zero. Moreover, for every $x \in \ell_{1}^{(n)}$ we can find $y \sim x$ as in Lemma 1 and since $y^{(j)}$ have mutually disjoint support, $F_{k_{1} \ldots k_{n}}(y)=0$ if for some two different numbers $i$ and $l, k_{i} \neq 0$ and $k_{l} \neq 0$. So $P(y)$ can be represented as an algebraic span of $F_{k}^{(j)}(y)$, $1 \leq j \leq n, k \in \mathbb{N}$. But $P(x)=P(y)$, and $F_{k}^{(j)}(x)=F_{k}^{(j)}(y)$. Thus $P$ is an algebraic span of separately symmetric polynomials $F_{k}^{(j)}(y), 1 \leq j \leq n, k \in \mathbb{N}$. Also, it is easy to check that $F_{k}^{(j)}$ are algebraically independent. So they form an algebraic basis in $\mathcal{P}_{s s}\left(\ell_{1}^{(n)}\right)$.

Let us denote by $H_{b s s}\left(\ell_{1}^{(n)}\right)$ the completion of $\mathcal{P}_{s s}\left(\ell_{1}^{(n)}\right)$ with respect to the topology of uniform convergence on bounded subsets of $\ell_{1}^{(n)}$. Clearly, $H_{b s s}\left(\ell_{1}^{(n)}\right)$ is a uniform Fréchet algebra and $H_{b s s}\left(\ell_{1}^{(n)}\right)$ is a closed subalgebra of the algebra $H_{b}\left(\ell_{1}^{(n)}\right)$ of entire functions of bounded type on $\ell_{1}^{(n)}$.

Let us recall that the spectrum of a Fréchet algebra is the set of all nonzero continuous complex homomorphisms. It is well known that the spectrum of algebra $H\left(\mathbb{C}^{n}\right)$ of entire functions on $\mathbb{C}^{n}$ consists of point-evaluation functionals $\delta_{z}, z \in \mathbb{C}^{n}, \delta_{z}(f)=f(z)$. Spectra of algebras $H_{b}(X)$ of entire functions on Banach spaces $X$ are much more complicated. In particular, in [2] it was shown that the spectra contain (but not limited to) point-evaluation functionals at elements of second dual spaces $X^{\prime \prime}$ of $X$. In [19] the spectra was described using second dual spaces to symmetric projective tensor products of $X$. Spectra of algebras of symmetric functions on $\ell_{p}$ were investigated in [1], [5], [6], [7]. It was shown that if $x, y \in \ell_{p}$ and $x=\sigma(y)$ for some permutation $\sigma$, then $\delta_{x}=\delta_{y}$. On the other hand, the spectrum of $H_{b s}\left(\ell_{p}\right)$ contains a family of elements $\psi_{\lambda}, \lambda \in \mathbb{C}$ which does not belong to the set of pointevaluation functionals. We denote by $M_{b s s}^{(n)}$ the spectrum of $H_{b s s}\left(\ell_{1}^{(n)}\right)$.

Theorem 2. The spectrum $M_{b s s}^{(n)}$ of $H_{b s s}\left(\ell_{1}^{(n)}\right)$ is the Cartesian product of $n$ copies of the spectrum $H_{b s}\left(\ell_{1}\right)$.

Proof. Let $\varphi$ be a continuous complex homomorphism of $H_{b s s}\left(\ell_{1}^{(n)}\right)$. Since $\left\{F_{k}^{(j)}\right\}$ forms an algebraic basis in $H_{b s s}\left(\ell_{1}^{(n)}\right), \varphi$ is completely defined by its values on the basis elements,

$$
\xi_{k}^{(j)}=\varphi\left(F_{k}^{(j)}\right), \quad j=1, \ldots, n, \quad k=1,2, \ldots
$$

For any fixed $j$, formula (5) defines a complex homomorphism $\varphi^{(j)}$ on $H_{b s}\left(\ell_{1}\right)$. So $\varphi$ is defined by the collection $\left(\varphi^{(1)}, \ldots, \varphi^{(n)}\right)$. Conversely, every such a collection uniquely defines an element in $M_{b s s}^{(n)}$.

Now let us consider the case of infinite direct sums. Recall that a sequence $\left\{e_{n}\right\}_{n=1}^{\infty}$ of a Banach space $X$ is called an unconditional (linear topological) basis if it is linearly independent and every $a \in X$ can be represented by

$$
a=\sum_{n=1}^{\infty} a_{n} e_{n}
$$


such that the series converges unconditionally.

For a given Banach space $X$ with an unconditional basis $\left\{e_{n}\right\}_{n=1}^{\infty}$ we consider a Banach space $\ell_{1}^{(X)}$ defined by the following way. If $x \in \ell_{1}^{(X)}$, then

$$
x=\left(x^{(1)}, x^{(2)}, \ldots, x^{(n)}, \ldots\right),
$$

where each $x^{(n)}=\left(x_{1}^{(n)}, \ldots, x_{k}^{(n)}, \ldots\right) \in \ell_{1}$ and

$$
\sum_{n=1}^{\infty}\left\|x^{(n)}\right\|_{\ell_{1}} e_{n} \in X
$$

with

$$
\|x\|=\left\|\sum_{n=1}^{\infty}\right\| x^{(n)}\left\|_{\ell_{1}} e_{n}\right\|_{X}
$$

Note that if $X=\ell_{1}$, then $\ell_{1}^{\left(\ell_{1}\right)}$ is isometrically isomorphic to the complete projective tensor product $\ell_{1} \widehat{\otimes} \ell_{1}$.

A polynomial $P$ on $\ell_{1}^{(X)}$ is separately symmetric if for every sequence of permutations on $\mathbb{N}, \sigma=\left(\sigma_{1}, \ldots, \sigma_{n}, \ldots\right)$ we have $P(\sigma(x))=P\left(\sigma_{1}\left(x^{(1)}\right), \ldots, \sigma_{n}\left(x^{(n)}\right), \ldots\right)=P(x)$ for all $x \in \ell_{1}^{(X)}$. Clearly, polynomials

$$
F_{m}^{(j)}(x)=\sum_{k=1}^{\infty}\left(x_{k}^{(j)}\right)^{m}, \quad j, m=1,2, \ldots
$$

are separately symmetric and algebraically independent. However, they does not form an algebraic basis in the algebra of all separately symmetric polynomials $\mathcal{P}_{s s}\left(\ell_{1}^{(X)}\right)$ in the general case.

Lemma 2. For every $a \in X, a=\sum_{j=1}^{\infty} a_{j} e_{j}$ there is a continuous complex homomorphism $\psi_{a}$ on $H_{b s s}\left(\ell_{1}^{(X)}\right)$ such that $\psi_{a}\left(F_{1}^{(j)}\right)=a_{j}$ and $\psi_{a}\left(F_{m}^{(j)}\right)=0$ for all $m>1$ and $j \in \mathbb{N}$.

Proof. Using an idea from [1], we define a sequence $v_{n} \in \ell_{1}^{(X)}$ by the following way

$$
v_{n}=\sum_{j=1}^{\infty}\left(v^{(j)}\right)_{n} e_{j}
$$

where $\left(v^{(j)}\right)_{n}$ belongs to the $j$ th copy of $\ell_{1}$ and

$$
\left(v^{(j)}\right)_{n}=(\underbrace{\frac{a_{j}}{n}, \ldots, \frac{a_{j}}{n}}_{n \text { times }}, 0,0, \ldots) .
$$

Then

$$
\left\|v_{n}\right\|=\left\|\sum_{j=1}^{\infty}\right\|\left(v^{(j)}\right)_{n}\left\|_{\ell_{1}} e_{j}\right\|_{X}=\left\|\sum_{j=1}^{\infty}\left|a_{j}\right| e_{j}\right\|_{X} \leq 2 K\left\|\sum_{j=1}^{\infty} a_{j} e_{j}\right\|_{X}=2 K\|a\|_{X},
$$

where $K$ is the unconditional constant of the basis $\left\{e_{j}\right\}_{j=1}^{\infty}$ (see [16, p. 19]). So the sequence $\left\{v_{n}\right\}_{n=1}^{\infty}$ is bounded. Since bounded subsets of spectra of Fréchet algebras are relatively compact, the corresponding sequence of point-evaluation complex homomorphisms $\left\{\delta_{v_{n}}\right\}_{n=1}^{\infty}$ must have a cluster point $\psi_{a}$. Since $F_{1}^{(j)}\left(v_{n}\right)=a_{j}$ for every $n \in \mathbb{N}, \psi_{a}\left(F_{1}^{(j)}\right)=a_{j}$. On the other hand, $F_{m}^{(j)}\left(v_{n}\right)=\frac{a_{j}^{m}}{n^{m-1}} \rightarrow 0$ as $n \rightarrow \infty$ and so $\psi_{a}\left(F_{m}^{(j)}\right)=0$ for all $m>1$ and $j \in \mathbb{N}$. 
We denote by $M_{b s s}^{(X)}$ the spectrum of $H_{b s s}\left(\ell_{1}^{(X)}\right)$. Let $\Psi: X \rightarrow H_{b s s}\left(\ell_{1}^{(X)}\right), \Psi(a)=\psi_{a}$.

Theorem 3. There is a homomorphism $C_{\Psi}$ from $H_{b s s}\left(\ell_{1}^{(X)}\right)$ onto $H_{b}(X)$ defined by

$$
C_{\Psi}(f)=\hat{f} \circ \Psi
$$

where $\hat{f}$ is the Gelfand transform of $f \in H_{b s s}\left(\ell_{1}^{(X)}\right)$.

Proof. Let $g(a)=C_{\Psi}(f)=\hat{f} \circ \Psi(a)=\psi_{a}(f), a \in X$. That is, $g$ is well defined on $X$. If $P_{n}$ is an $n$-homogeneous polynomial on $X$, then $C_{\Psi}\left(P_{n}\right)$ is an $n$-homogeneous polynomial on $X$ and

$$
\left|C_{\Psi}\left(P_{n}\right)(a)\right| \leq \sup _{m}\left|P_{n}\left(v_{m}\right)\right| \leq\left\|P_{n}\right\| \sup _{m}\left\|v_{m}\right\|^{n}=\left\|P_{n}\right\|\left\|\sum_{j=1}^{\infty}\left|a_{j}\right| e_{j}\right\|_{X}^{n} \leq\left\|P_{n}\right\|(2 K)^{n}\|a\|_{X}^{n} .
$$

Thus $\left\|C_{\Psi}\left(P_{n}\right)\right\| \leq(2 K)^{n}\left\|P_{n}\right\|$, where $K$ is the unconditional constant of the topological basis in $X$. So

$$
g(a)=\sum_{n=0}^{\infty} C_{\Psi}\left(P_{n}\right)(a)
$$

where $P_{n}$ are like in (1) and according to $(2), \varrho_{0}(g)=\frac{1}{2 K} \varrho_{0}(f)=\infty$. So $g \in H_{b}(X)$.

Conversely, let $g \in H_{b}(X)$ and

$$
g(a)=\sum_{n=0}^{\infty} Q_{n}(a)
$$

be the representation of $g$ by the series of homogeneous polynomials. Since $X$ has an unconditional basis, for $n>0$

$$
Q_{n}(a)=Q_{n}\left(\sum_{m=1}^{\infty} a_{m} e_{m}\right)=\sum_{i_{1}, \ldots, i_{n}=1}^{\infty} c_{i_{1}, \ldots, i_{n}} a_{i_{1}} \cdots a_{i_{n}}
$$

for some constants $c_{i_{1}, \ldots, i_{n}}$. We set $P_{0}=Q_{0}$ and

$$
P_{n}(x)=\sum_{i_{1}, \ldots, i_{n}=1}^{\infty} c_{i_{1}, \ldots, i_{n}} F_{1}^{\left(i_{1}\right)}(x) \cdots F_{1}^{\left(i_{n}\right)}(x) .
$$

Since $\left\|F_{1}^{(j)}\right\|=1,\left|F_{1}^{(j)}(x)\right| \leq\left\|x^{(j)}\right\|$ and so $\left\|P_{n}\right\| \leq\left\|Q_{n}\right\|$. Thus if we define

$$
f(x)=\sum_{n=0}^{\infty} P_{n}(x), \quad x \in \ell_{1}^{(X)},
$$

then $\varrho_{0}(f) \geq \varrho_{0}(g)=\infty$ and so $f \in H_{b s s}\left(\ell_{1}^{(X)}\right)$. According to the definition of $\Psi$ and Lemma $2, C_{\Psi}(f)=g$ and so $C_{\Psi}$ is onto.

Note that in the general case $H_{b}(X)$ is nonseparable and so $H_{b s s}\left(\ell_{1}^{(X)}\right)$ is nonseparable and can not to be generated by a countable algebraic basis.

Corollary 1. The spectrum $M_{b s s}^{(X)}$ contains a copy of the spectrum of $H_{b}(X)$, in particular, it contains a point-wise copy of $X^{\prime \prime}$. 


\section{REFERENCES}

1. R. Alencar, R. Aron, P. Galindo, A. Zagorodnyuk, Algebras of symmetric holomorphic functions on $\ell_{p}$, Bull. Lond. Math. Soc., 35 (2003), 55-64.

2. R.M. Aron, B.J. Cole, T.W. Gamelin, Spectra of algebras of analytic functions on a Banach space, J. Reine Angew. Math., 415 (1991), 51-93.

3. R.M. Aron, J. Falcó, D. García, M. Maestre, Algebras of symmetric holomorphic functions of several complex variables. Rev. Mat. Complut., 31 (2018), 651-672.

4. R. Aron, P. Galindo, D. Pinasco, I. Zalduendo, Group-symmetric holomorphic functions on a Banach space. Bull. Lond. Math. Soc., 48 (2016), №5, 779-796.

5. I. Chernega, P. Galindo, A. Zagorodnyuk, Some algebras of symmetric analytic functions and their spectra. Proc. Edinburgh Math. Soc., 55 (2012), 125-142.

6. I. Chernega, P. Galindo, A. Zagorodnyuk, The convolution operation on the spectra of algebras of symmetric analytic functions, J. Math. Anal. Appl., 395 (2012), 569-577.

7. I.Chernega, P. Galindo, A. Zagorodnyuk, A multiplicative convolution on the spectra of algebras of symmetric analytic functions, Rev. Mat. Complut., 27 (2014), №2, 575-585.

8. M. González, R. Gonzalo, J. Jaramillo, Symmetric polynomials on rearrangement invariant function spaces, J. London Math. Soc., 59 (1999), №2, 681-697.

9. S. Dineen, Complex Analysis in Infinite Dimensional Spaces. London: Springer, 1999.

10. P. Galindo, T. Vasylyshyn, A. Zagorodnyuk, The algebra of symmetric analytic functions on $L_{\infty}$, Proc. R. Soc. Edinb., Sect. A, Math., 147 (2017), №4, 743-761.

11. P. Galindo, T. Vasylyshyn, A. Zagorodnyuk, Symmetric and finitely symmetric polynomials on the spaces $\ell_{\infty}$ and $L_{\infty}[0,+\infty)$, Mathematische Nachrichten, 291 (2018), 1712-1726.

12. P. A. MacMahon, Combinatory analysis, Chelsea Publishing Co. New York, 1960.

13. V. Kravtsiv, T. Vasylyshyn, A. Zagorodnyuk, On algebraic basis of the algebra of symmetric polynomials on $\ell_{p}\left(\mathbb{C}^{n}\right)$, Journal of Function Spaces, 7 (2017), Article ID 4947925, 8 p.

14. V. V. Kravtsiv, A. V. Zagorodnyuk, Representation of spectra of algebras of block-symmetric analytic functions of bounded type, Carpath. Math. Publications, 8 (2016), №2, 263-271.

15. V. V. Kravtsiv, A. V. Zagorodnyuk, On algebraic bases of algebras of block-symmetric polynomials on Banach spaces, Mat. Stud., 37 (2012), №1, 109-112.

16. J. Lindestrauss, L. Tzafriri, Classical Banach spaces I. Sequence Spaces, Springer-Verlag, New York, 1977. $190 \mathrm{p}$.

17. J. Mujica, Complex Analysis in Banach Spaces, North-Holland, Amsterdam, New York, Oxford, 1986.

18. T. Vasylyshyn, Topology on the spectrum of the algebra of entire symmetric functions of bounded type on the complex $L_{\infty}$. Carpathian Math. Publ., 9 (2017), №1, 22-27.

19. A. Zagorodnyuk, Spectra of algebras of entire functions on Banach spaces, Proc. Amer. Math. Soc., 134 (2006), 2559-2569.

Vasyl Stefanyk Precarpathian National University

Ivano-Frankivsk, Ukraine

farah.jawad@yahoo.com

Received 30.10.2018

Revised 12.12.2018 